\title{
Family- and school-based correlates of energy balance-related behaviours in 10-12-year-old children: a systematic review within the ENERGY (EuropeaN Energy balance Research to prevent excessive weight Gain among Youth) project
}

\author{
Maïté Verloigne ${ }^{1, *}$, Wendy Van Lippevelde ${ }^{2}$, Lea Maes ${ }^{2}$, Johannes Brug ${ }^{3}$ and \\ Ilse De Bourdeaudhuij ${ }^{1}$ \\ 'Department of Movement and Sport Sciences, Ghent University, Watersportlaan 2, B-9000 Ghent, Belgium: \\ ${ }^{2}$ Department of Public Health, Ghent University, Ghent, Belgium: ${ }^{3}$ Department of Epidemiology \& Biostatistics \\ and the EMGO Institute for Health and Care Research, VU University Medical Center, Amsterdam, \\ The Netherlands
}

Submitted 11 April 2011: Accepted 27 October 2011: First published online 24 January 2012

\begin{abstract}
Objective: To identify family- and school-based correlates of specific energy balance-related behaviours (physical activity, sedentary behaviour, breakfast consumption, soft drink consumption) among 10-12-year-olds, using the EnRG framework (Environmental Research framework for weight Gain prevention).

Design: A literature review to identify observational studies exploring at least one family- or school-based correlate of the specific behaviours, resulting in seventysix articles.

Setting: Eighteen studies were conducted in Europe, forty-one studies in North America and seventeen studies in Australasia.

Subjects: Healthy children aged 10-12 years.

Results: Parental and maternal physical activity, doing physical activities with parents and parental logistic support were identified as the most important, positive correlates of physical activity. Parental rules was the most important correlate of sedentary behaviour and was inversely related to it. School socioeconomic status was positively related to physical activity and inversely related to sedentary behaviour. The available studies suggested a positive relationship between soft drink availability at home and consumption. Soft drink availability and consumption at school were the most important school-based correlates of soft drink consumption. A permissive parenting style was related to more soft drink consumption and less breakfast consumption.

Conclusions: An important role has been awarded to parents, suggesting parents should be involved in obesity prevention programmes. Despite the opportunities a school can offer, little research has been done to identify school-environmental correlates of energy balance-related behaviours in this age group. Obesity prevention programmes can focus on the most important correlates to maximize the effectiveness of the programme. Future research should aim at longitudinal studies.
\end{abstract}

Overweight and obesity are highly prevalent among children and are associated with several childhood and further lifecourse physical and psychological problems ${ }^{(1,2)}$. Subsequently, there is an urgent need to develop effective obesity prevention strategies for children.

Overweight and obesity are caused by a lasting positive energy imbalance ${ }^{(3)}$. Because energy intake and expenditure mainly result from specific dietary and physical activity (PA) behaviours, a first step in the development of an obesity prevention programme is to identify these behaviours associated with unnecessary weight gain. The next essential step is to identify the behavioural correlates of these specific energy balance-related behaviours (EBRB) that can be targeted in intervention programmes ${ }^{(4-6)}$. The 'EuropeaN Energy balance Research to prevent excessive weight Gain among Youth' (ENERGY) project aims to develop a theoryand evidence-based intervention programme to prevent unnecessary weight gain among children ${ }^{(7)}$. One of the objectives of the ENERGY project is therefore to identify the most important correlates of EBRB via a systematic review.

A number of reviews have summarized the available evidence regarding correlates of EBRB in children and 
adolescents $^{(8-16)}$. These reviews have focused on a wide age range, mostly capturing 6-18-year-olds. However, children in the transition from childhood to adolescence gain more autonomy and decision-making power regarding PA and dietary behaviours ${ }^{(17)}$, making this a critical period for changes in health behaviour ${ }^{(18,19)}$. Studies show that in this age group children start receiving pocket money that they may use for food purchases, for example, and they have more meals without parental presence ${ }^{(17)}$. Additionally, these children prepare for or will go from primary school to secondary school: a different school environment characterized by a higher likelihood of presence of vending machines and school shops, and with different food and PA policies ${ }^{(20,21)}$. Finally, a steep increase in the prevalence of overweight and obesity is observed in this age group ${ }^{(22)}$. Because of the specificity of the age group, it is important to gain more insight into the potential drivers of relevant EBRB among 10-12-year-olds. This enables researchers to target the correlates of EBRB specifically for 10-12-year-olds when developing an obesity prevention programme for this age group. Despite the gradually growing independence regarding dietary and PA behaviour choices in this age group, the family environment is still most likely to be of major importance in influencing children's EBRB through a variety of mechanisms such as parental modelling behaviour, encouragement and practices ${ }^{(17,23)}$. Parents determine both the physical and social environment of their children ${ }^{(17)}$, suggesting effective obesity prevention programmes must consider the family as an intervention target ${ }^{(24)}$. Also the school plays a significant role, since schools have the capacity to offer various opportunities to practise healthy dietary behaviours and to engage in $\mathrm{PA}^{(25,26)}$. Moreover, the majority of children (including lower social classes) can be easily accessed through schools and children spend a significant amount of their time in schools. A better understanding of the family- and school-based correlates of PA and dietary behaviours in children will add to better informed obesity prevention programmes. Finally, previous reviews have focused mainly on one or two specific EBRB, but EBRB and their correlates should be studied within an energy balance approach; that is, focusing on energy input as well as output ${ }^{(6)}$. The present review therefore investigates correlates of several EBRB to focus on both energy intake and expenditure. The EBRB in the current review are PA, sedentary behaviour, breakfast and soft drink consumption. Previous studies and reviews have provided evidence that PA and breakfast consumption are related to overweight and obesity in children ${ }^{(27-29)}$. For sedentary behaviour and soft drink consumption, the evidence is inconsistent ${ }^{(30-33)}$ and further research is needed to reveal the mechanism between these behaviours and obesity. However, a review conducted as part of the ENERGY project showed that most evidence for an association with overweight and obesity in 10-12-year-old children was found for these four $\mathrm{EBRB}^{(34)}$. Therefore, the present review focuses on correlates of PA, sedentary behaviour, breakfast and soft drink consumption.

A theoretical approach is needed to get insight into the complexity of correlates that are related to EBRB ${ }^{(35)}$. Kremers et al. ${ }^{(36)}$ have proposed the Environmental Research framework for weight Gain prevention (EnRG framework), which integrates potential personal psychological correlates, referred to as 'cognitive' factors in the model, with environmental factors (adopted from the ANalysis Grid for Environments Linked to Obesity (ANGELO) framework) and identifies important moderators, including personal and behavioural factors, to gain insight into the processes that underlie EBRB. Environmental factors can have a direct impact on EBRB or can be mediated by the personal psychological factors. In the ENERGY project, the EnRG framework is adopted with a specific focus on the family and school environment ${ }^{(7)}$. The specific focus on family and school is important, as the ENERGY project aims to develop a family-involving, school-based intervention to prevent overweight.

In brief, the objective of the present systematic review was to identify family- and school-based correlates of PA, sedentary behaviour, breakfast consumption and soft drink consumption in 10-12-year-old children. The EnRG framework was used to inform the ENERGY project on the most important correlates.

\section{Methods}

\section{Search strategy}

Medline (PubMed), Web of Science, CINAHL and The Cochrane Library electronic databases were searched from 1990 to September 2010. The search strategy described population, study design, context, predictor variables and outcome behaviour. Only English-language published articles were located. The full search strategy is described in online Appendix A.

\section{Inclusion criteria}

To be included, studies had to meet all of the following inclusion criteria: (i) studies were limited to samples comprising healthy 10-12-year-old children (mean age: 9.5-12.5 years); (ii) only observational studies were included, whereas dissertations and studies investigating interventions or with a quasi-experimental design were excluded (with the exception of studies reporting on baseline data from intervention studies); and (iii) studies had to examine at least one family- or school-based correlate of PA, sedentary behaviour, breakfast or soft drink consumption.

\section{Selection process}

A first selection was made by screening titles and abstracts by the first author using the aforementioned 
inclusion criteria. After screening the full text of those articles, a final selection of articles to be included was made. Additionally, reference lists of the retrieved articles and review articles were checked for additional relevant papers.

\section{Data extraction}

Relevant data on author, date, study design, sample size, participants' age, study context, outcome measures, instruments and the examined correlates from the included studies were extracted into detailed summary tables. Information on the studies' characteristics were summarized (see Results).

\section{Categorization of variables}

The categorization of the correlates was based on the EnRG framework which includes three main groups: environment, personal psychological mediators and moderators ${ }^{(36)}$. Since we specifically focused on family- and school-based correlates only environmental factors were included, divided into family- and school-environmental factors. For a further classification of the variables, the 'types' of environments according to the ANGELO framework - i.e. one of the key inputs for EnRG - was used ${ }^{(37)}$. Table 1 provides an overview of all category definitions. The EnRG framework, adopted for the ENERGY project, is described elsewhere ${ }^{(7)}$. Data were summarized into four tables which give an overview of all family- and school-environmental correlates for PA, sedentary behaviour, breakfast and soft drink consumption, respectively (Tables 3-6). Longitudinal studies were highlighted in bold. Previous studies have solely included correlates examined in at least three studies. Because of the limited amount of studies examining correlates of the four EBRB in 10-12-year-olds, all studied correlates were taken into consideration. This enabled us to identify all variables that have already been investigated for this age group and to provide a comprehensive overview of the correlates by means of a table. Finally, it must be taken into account that one article can investigate a correlate several times, for example when the article investigated correlates of total PA, moderate PA and vigorous PA separately. In that case, the study number was listed three times in the table, since the association between the correlate and the EBRB has basically been investigated three times. Conceptually similar variables were combined for consistency of interpretation, resulting again in the possibility of one article listed multiple times for one correlate. All correlates and their range of definitions are described in online Appendices B, C, D and E.

\section{Coding and summarizing associations}

The coding of results was similar to previous reviews $(8,9,14,16,38)$ and is also explained in the footnotes to Tables 3-6, where each studied correlate received a final summary association code: no association, an indeterminate association or a positive or negative association. As a consequence of the diversity of variables, samples, measures and analyses in the retrieved studies, we have focused on the consistency of the association and not on the strength of the association. If analyses were conducted separately for male and female participants, 'M' or 'F' was indicated. If analyses were conducted for different time periods (e.g. follow-up of 1 and 2 years), 'I' and 'II' were indicated.

\section{Results}

\section{Papers retrieved}

The search for articles in the four databases resulted in 13258 articles. Based on titles and abstracts, the full text of 316 potentially relevant articles was retrieved and reviewed. This resulted in a total of sixty-six articles that met all inclusion criteria. Another ten articles were included based on the reference lists of retrieved articles and reviews, which brought the final number to seventysix articles (Fig. 1).

\section{General characteristics of the studies reviewed}

Table 2 gives an overview of the characteristics of the studies reviewed. In brief, the majority of articles were cross-sectional (fifty-seven studies); eighteen studies (24\%) were conducted in Europe, forty-one studies (54\%) in North America and seventeen studies (22\%) in Australasia.

Table 1 Categorization of the variables

\begin{tabular}{ll}
\hline Category & Definition \\
\hline $\begin{array}{l}\text { Family environment* } \\
\text { School environment }\end{array}$ & $\begin{array}{l}\text { The micro-environmental setting of the home and family environment } \\
\text { Physical environmentt }\end{array}$ \\
$\begin{array}{l}\text { The micro-environmental setting of the school } \\
\text { Sociocultural environmentt }\end{array}$ & $\begin{array}{l}\text { Availability and accessibility of dietary, PA and sedentary behavioural choices } \\
\text { Factors regarding what is socially appropriate, acceptable or desirable as related to } \\
\text { dietary, PA and sedentary behavioural choices }\end{array}$ \\
Economic environmentt & $\begin{array}{r}\text { Factors related to the 'affordability' of dietary, PA and sedentary behavioural choices, } \\
\text { i.e. financial opportunities regarding, and the costs of, dietary, PA and sedentary } \\
\text { behavioural choices } \\
\text { Rules and regulations regarding dietary, PA and sedentary behavioural choices }\end{array}$ \\
\hline
\end{tabular}

PA, physical activity.

*EnRG (Environmental Research framework for weight Gain prevention) framework, adopted for the ENERGY (EuropeaN Energy balance Research to prevent excessive weight Gain among Youth) project.

tANGELO (ANalysis Grid for Environments Linked to Obesity) framework. 


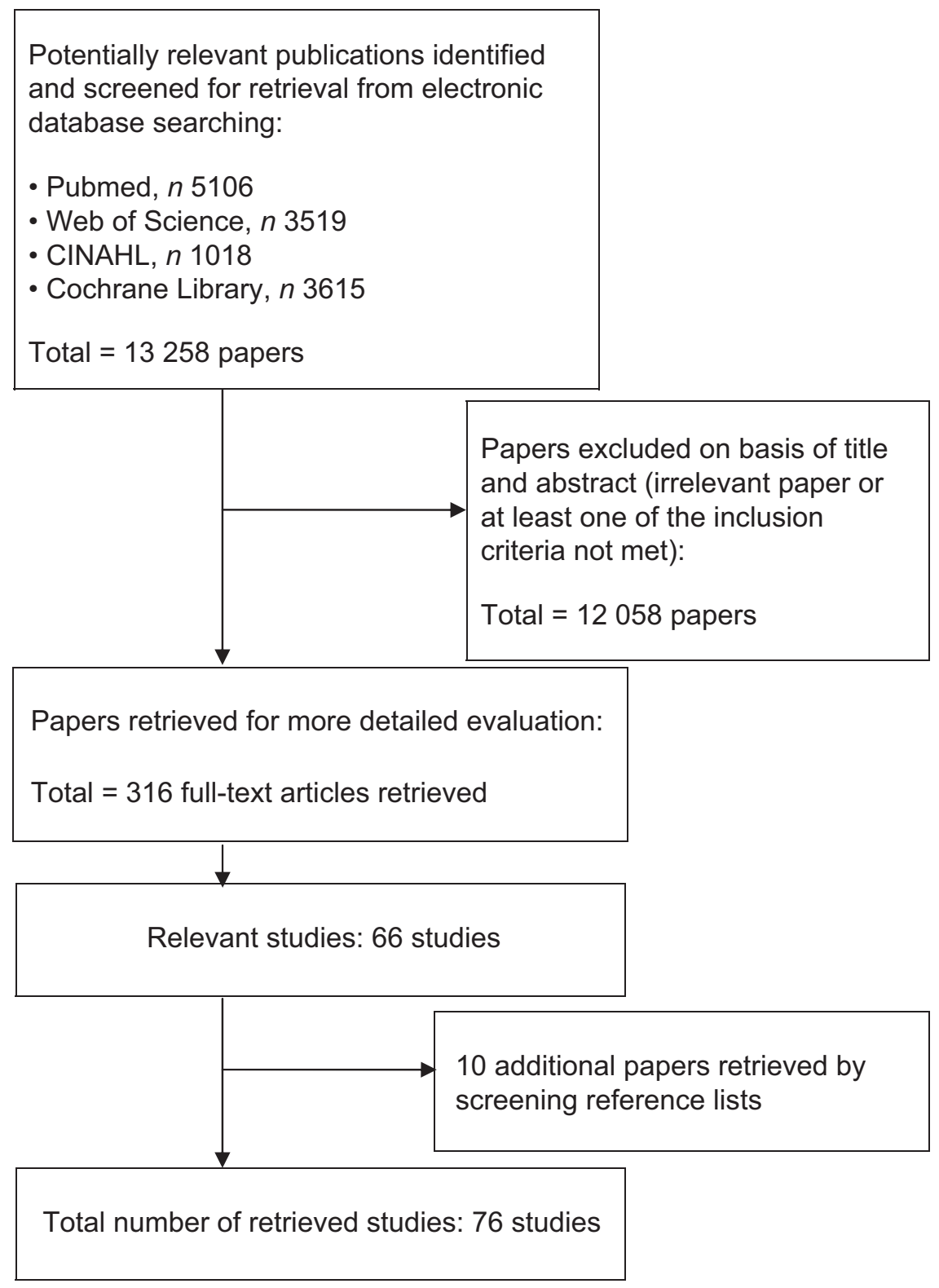

Fig. 1 Flowchart of the study selection process

Sample size ranged from thirty-eight to 16202 . Correlates of PA were studied the most (fifty-five studies).

\section{Correlates of energy balance-related behaviours}

Table 3 gives an overview of all correlates found for PA, Table 4 for sedentary behaviour, Table 5 for breakfast consumption and Table 6 for soft drink consumption.

Physical activity behaviour (Table 3)

Family-environmental variables. Thirty-eight familyenvironmental variables were studied for PA: four physical, twenty-seven sociocultural, four economic and three political environmental variables. Most evidence was found for parental/family PA, maternal PA, doing physical activities with the parents and logistic support. Other positive associations with PA were found for home equipment/ opportunities for sedentary behaviour, sedentary time with parents, parental beliefs towards screen-based behaviours and parental enjoyment of screen-based behaviours. Parental sedentary time, parental enjoyment of PA, parental barriers, parental self-efficacy and parental rules/restriction regarding screen-based behaviours were inversely associated with PA. All other variables showed an indeterminate association or no association with PA.

School-environmental variables. Twelve schoolenvironmental variables were studied: six physical, three sociocultural, one economic and two political environmental variables. Walking to and from school, teacher support and school socio-economic status (SES) were positively associated with PA. Having class problems was inversely 
Table 2 General characteristics of the studies reviewed

\begin{tabular}{|c|c|}
\hline & Bibliography no. \\
\hline \multicolumn{2}{|l|}{ Sample size } \\
\hline$<100$ & $15,51,75$ \\
\hline $100-199$ & $4,9,17,28,37,43,59,60,70$ \\
\hline 200-299 & $11,23,25,26,61,69$ \\
\hline $300-499$ & $2,10,13,19,20,36,39,48,49,54$ \\
\hline 500-999 & $1,5,6,14,16,18,22,29,30,33,52,53,55,56,57,58,65$ \\
\hline $1000-2999$ & $3,12,32,35,38,40,42,45,46,47,62,63,64,66,67,68,72,73,74,76$ \\
\hline $3000-4999$ & $7,31,41,44,71$ \\
\hline$>5000$ & $8,21,24,27,34,50$ \\
\hline \multicolumn{2}{|l|}{ Study design } \\
\hline Cross-sectional & $\begin{array}{l}1,5,6,7,8,9,10,16,19,21,22,23,24,25,27,28,29,30,31,32,33,36,37,39,40,41 \\
\quad 46,49,42,43,44,45,47,48,50,51,53,54,55,56,57,58,59,60,63,64,66,67,68 \\
\quad 69,70,71,72,73,74,75,76\end{array}$ \\
\hline Longitudinal & $\begin{array}{l}2(+3 y), 3(+1 y,+2 y), 4(+1 y), 11(+5 y), 12(+20 y), 13(+5 y), 14(+1 y), 15(+2 y,+4 y) \\
17(+3 y), 18(+2 y), 20(+5 y), 26(+3 y), 34(+1 y,+8 y), 35(+4 y,+7 y), 38(+2 y), 52 \\
\quad(+20 \mathrm{~m}), 61(+1 y), 62(+1 y), 65(+3 y)\end{array}$ \\
\hline \multicolumn{2}{|r|}{ (+ 200111$), 01(+1 y), 0<(+1 y), 05$ (+3y) } \\
\hline Europe & $4,7,23,26,36,39,40,41,46,47,49,50,65,66,67,68,71,76$ \\
\hline North America & $\begin{array}{l}3,5,6,8,15,16,17,18,20,21,22,25,27,28,29,30,31,34,35,37,38,42,43,44,45 \\
\quad 48,51,52,54,55,56,59,60,61,62,69,70,72,73,74,75\end{array}$ \\
\hline Australasia & $1,2,9,10,11,12,13,14,19,24,32,33,53,57,58,63,64$ \\
\hline \multicolumn{2}{|r|}{$1,2,9,10,11,12,10,14,19,24,02,00,30,31,30,00,04$} \\
\hline PA & $\begin{array}{l}1,2,3,4,7,9,11,12,13,15,17,18,19,20,22,23,25,26,28,31,32,33,34,35,36,37 \\
\quad 38,39,42,43,45,46,47,48,49,50,51,52,53,54,55,57,58,59,60,61,62,65,66 \\
\quad 68,69,71,72,75,76\end{array}$ \\
\hline Sedentary behaviour & $2,7,8,30,37,51,53,55,56,64,71,74,76$ \\
\hline Breakfast consumption & $10,24,40,41,63,67,76$ \\
\hline Soft drink consumption & $5,6,14,16,21,27,29,44,67,70,73$ \\
\hline \multicolumn{2}{|l|}{ Gender } \\
\hline Boys and girls combined & $\begin{array}{c}4,5,6,8,10,14,16,20,21,22,24,25,27,28,29,30,31,32,33,37,39,40,41,42,44 \\
46,47,48,49,50,54,56,62,63,64,66,67,68,72,73,74,75,76\end{array}$ \\
\hline Boys and girls reported separately & $1,2,3,7,9,11,12,13,17,19,23,26,34,36,45,51,52,53,57,58,59,60,61,65,71$ \\
\hline Girls only & $15,18,35,38,43,55,69,70$ \\
\hline \multicolumn{2}{|l|}{ Date of the study } \\
\hline Before 2000 & $\begin{array}{l}3,7,12,14,15,17,21,23,25,26,27,29,34,35,36,37,38,39,45,48,52,54,59,60 \text {, } \\
\quad 61,65,72,74\end{array}$ \\
\hline 2000 or after & $\begin{array}{c}1,2,4,5,6,8,9,10,11,13,16,18,19,20,22,24,28,30,31,32,33,36,40,41,42,43 \\
44,46,47,49,50,51,53,55,56,57,58,62,63,64,66,67,68,69,70,71,73,76\end{array}$ \\
\hline \multicolumn{2}{|r|}{ (1, } \\
\hline Child report & $\begin{array}{r}3,5,6,7,8,10,14,16,17,19,21,23,24,25,26,27,29,30,31,33,34,35,38,40,41,42,43 \\
44,45,46,47,48,49,54,55,56,61,62,63,64,65,67,68,69,70,71,72,73,74,75,76\end{array}$ \\
\hline Parent report & $11,57,58$ \\
\hline Objective measurement & $1,9,13,15,18,36,39,50,59,60,66$ \\
\hline Parent report + child report & $4,22,32,37$ \\
\hline Child report + objective measurement & $2,12,20,28,51$ \\
\hline Parent report + objective measurement & 53 \\
\hline $\begin{array}{l}\text { Parent report }+ \text { child report }+ \text { objective } \\
\text { measurement }\end{array}$ & 52 \\
\hline \multicolumn{2}{|l|}{ Reliability/validity of child and parent reports } \\
\hline Unknown/Not reported/Poor & $8,16,21,22,26,29,37,42,46,47,56,63,64,73,76$ \\
\hline Acceptable & $\begin{array}{l}2,3,4,5,6,7,10,11,12,14,17,19,20,23,24,25,27,28,30,31,32,33,34,35,38,40,41, \\
\quad 43,44,45,48,49,51,52,53,54,55,57,58,61,62,65,67,68,69,70,71,72,74,75\end{array}$ \\
\hline \multicolumn{2}{|r|}{ 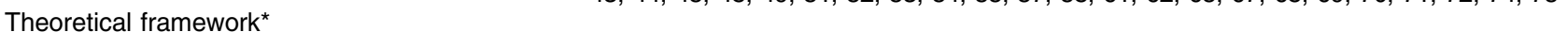 } \\
\hline Social Cognitive Theory ${ }^{(39)}$ & $16,18,20,41,48,52,54,59,60,61,69,70$ \\
\hline Theory of Reasoned Action ${ }^{(40)}$ & $41,48,54,60$ \\
\hline Social Learning Theory ${ }^{(41)}$ & 17,40 \\
\hline Youth PA Promotion Mode ${ }^{(42)}$ & 19,72 \\
\hline Family Influence Model ${ }^{(43)}$ & 43 \\
\hline $\begin{array}{l}\text { Social Determinants of Health and } \\
\text { Environmental Health } \\
\text { Promotion Model }{ }^{(44)}\end{array}$ & 22 \\
\hline Health Promotion Model ${ }^{(45)}$ & 25 \\
\hline Kohl and Hobbs' model ${ }^{(46)}$ & $7,31,37$ \\
\hline Socio-ecological models & $\begin{array}{l}11,29,38,39,42,46,55,56,57,47^{(47)} \\
13,53,58^{(48)} \\
30^{(49)}\end{array}$ \\
\hline Model of achievement-related choice ${ }^{(50)}$ & 4 \\
\hline Ferraro's model ${ }^{(51)}$ & 9 \\
\hline
\end{tabular}




\begin{tabular}{|c|c|}
\hline & Bibliography no. \\
\hline \multicolumn{2}{|l|}{ Specific PA behaviours } \\
\hline Total MPA & $2,2,22,43,60$ \\
\hline Total VPA & $2,2,7,17,22,37,4355,60$ \\
\hline Total MVPA & $3,4,9,13,15,18,19,34,50,51,55,59,62,66$ \\
\hline Total PA & $2,2,12,20,22,25,33,36,39,42,45,50,51,53,65,68,69,72,75,76$ \\
\hline Leisure-time VPA & 61 \\
\hline Leisure-time MVPA & 42,61 \\
\hline Leisure-time PA & $12,22,23,26,28,31,32,35,37,38,48,49,52,54,71$ \\
\hline Leisure-time: time spent outdoors & 11 \\
\hline MVPA at school & 42 \\
\hline Active transportation to school & $2,22,46,47,57,58$ \\
\hline Number of steps & 12,28 \\
\hline Number of steps at school & 1 \\
\hline Number of steps in weekend & 1 \\
\hline Sports participation & $22,23,28,37$ \\
\hline Participation in other organized PA/lessons & 22,31 \\
\hline \multicolumn{2}{|l|}{ Specific sedentary behaviours } \\
\hline TV & $2,37,51,56,64$ \\
\hline $\mathrm{TV}+$ computer + games & $7,8,30,53,55,74,76$ \\
\hline TV + computer + games + reading & 71 \\
\hline Time spent sedentary & 2 \\
\hline \multicolumn{2}{|l|}{ Recall period PA and sedentary behaviour } \\
\hline $1 \mathrm{~d}$ & $8,25,32,33,48,51,52,54,55,61,75,76$ \\
\hline $3 d$ & 17 \\
\hline $7 \mathrm{~d}$ & $3,4,7,19,20,22,30,31,43,45,62,64$ \\
\hline Past month & 49 \\
\hline Past year & $34,35,37,71$ \\
\hline Usual week (or other time period) & $2,11,23,26,28,38,42,46,47,53,56,57,58,65,68,69,72,74$ \\
\hline No recall (objective measurement) & $1,2,9,12,13,15,18,36,39,50,59,60,66$ \\
\hline \multicolumn{2}{|l|}{$\begin{array}{l}\text { Measurement instrument breakfast/soft drink } \\
\text { consumption }\end{array}$} \\
\hline $24 \mathrm{~h}$ recall & $5,6,40$ \\
\hline $7 \mathrm{~d}$ recall & $21,63,73$ \\
\hline $5 \mathrm{~d}$ food record & 14 \\
\hline FFQ & $27,67,70$ \\
\hline Other questionnaire & $10,16,24,29,41,44,76$ \\
\hline
\end{tabular}

PA, physical activity; MPA, moderate physical activity; VPA, vigorous physical activity; MVPA, moderate-to-vigorous physical activity, TV, television; $y$, year(s); m, months.

${ }^{\star}$ For theoretical frameworks, superscript number in parentheses refers to reference in the reference list.

associated. An inconsistent association with PA was found for participation in school sports (team). The other school environmental variables were not related to PA.

Regarding all studied correlates of PA, one remarkable finding was noticed. The studies that did not find an association between maternal and child PA were North American studies. In contrast, four European studies and one Australian study revealed a positive relationship between maternal and child PA. For all other correlates, no relevant differences were found between European, North American and Australasian results.

\section{Sedentary behaviour (Table 4)}

Family-environmental variables. Twenty-eight familyenvironmental variables were examined: four physical, eighteen sociocultural, five economic and one political environmental variable. Most evidence was found for a negative relationship between parental rules/restriction regarding screen-based behaviours and sedentary behaviour. Living in a two-parent household, parental ethnicity, parental PA preferences, parental knowledge about recom- mendations and having family dinners were negatively related to sedentary behaviour. A positive association was found for number of televisions (TV) in the household, eating in front of the TV, parental overweight, parental and maternal sedentary time, sedentary time with parents, parental enjoyment of screen-based behaviours, and household income. All other variables showed an indeterminate association or no association with sedentary behaviour.

School-environmental variables. Two schoolenvironmental variables were studied: one physical and one economic environmental variable. School SES was inversely associated with sedentary behaviour. The after-school context was not associated.

Generally, no relevant differences were found between European, North American and Australasian results. One study had a longitudinal design.

\section{Breakfast consumption (Table 5)}

Family-environmental variables. Fourteen familyenvironmental variables were studied: eleven sociocultural, two economic and one political environmental variable. 
Table 3 Correlates of physical activity behaviour in 10-12-year-old children: bibliography numbers of studies reporting a positive correlation (+), a negative correlation (-) or no correlation (0) among the studies reviewed

\begin{tabular}{|c|c|c|c|c|c|}
\hline Physical activity & + & - & 0 & $n / N$ & Assoc. code \\
\hline \multicolumn{6}{|l|}{ Family environment } \\
\hline \multicolumn{6}{|l|}{ Physical } \\
\hline Home equipment/opportunities for PA & $48,59(F)$ & & $11,59,60,61,61$ & $2 / 7$ & 00 \\
\hline Home equipment/opportunities for sedentary behaviour & $39,53(\mathrm{M})$ & $53(\mathrm{M})$ & & $2 / 3$ & + \\
\hline Access or ownership car & & 46 & 57 & $1 / 2$ & $?$ \\
\hline Having a dog & $58(\mathrm{M})$ & & 11 & $1 / 2$ & ? \\
\hline \multicolumn{6}{|l|}{ Sociocultural } \\
\hline Two-parent household & $71(\mathrm{M})$ & 37 & $\begin{array}{l}\text { 7, 20, 22, 22, 22, 22, 22, 22, 22, 26} \\
\quad 32,32,35,37,37,39,45,45,45 \\
\quad 49,52,57,76\end{array}$ & $1 / 25$ & 00 \\
\hline Number of family members & & & $45,45,45$ & $0 / 3$ & 0 \\
\hline Biological parents living at home & \multirow{2}{*}{\multicolumn{2}{|c|}{ 13(F), 71(F) }} & 39,39 & $0 / 2$ & 0 \\
\hline Number of siblings & & & $32,32,57$ & $2 / 5$ & ? \\
\hline Birth order & & & 32,32 & $0 / 2$ & 0 \\
\hline Parental ethnicity & \multicolumn{2}{|l|}{ 26(F), 32, 32} & $32,32,32,32,45,45,45,62$ & $3 / 11$ & 00 \\
\hline Parental overweight & & \multirow[t]{3}{*}{71} & 39,39 & $1 / 3$ & 0 \\
\hline Parental age & & & 39,39 & $0 / 2$ & 0 \\
\hline Parental/family $\mathrm{PA}^{*}$ & $\begin{array}{l}\text { 13(M), 28, 28, 28, 52(M), 71, 72, } \\
\quad 72,75\end{array}$ & & $20,28,28,28$ & $9 / 13$ & ++ \\
\hline Maternal PAt & $\begin{array}{l}\text { 4, 13(M), 38(I), 38(II), 39, 45, 45(F), } \\
\text { 46, 59(M), 60(M), 61(F), } 71\end{array}$ & 43,45 & $34,43,48,59,61$ & $12 / 19$ & ++ \\
\hline Paternal PA $\ddagger$ & $\begin{array}{l}\text { 13(F), 38(I), 38(II), 39(F), 45, } \\
\text { 59(M), 71(F) }\end{array}$ & 45 & $4,45,48,59,60,61,61$ & $7 / 15$ & ?? \\
\hline PA with parents & $\begin{array}{l}\text { 13(F), 31, 38(I), 38(II), 39, 52, } \\
\text { 52(M), 55, 55 }\end{array}$ & & 31 & 9/10 & ++ \\
\hline Parental sedentary time & & $53(\mathrm{M}), 53(\mathrm{~F})$ & & $2 / 2$ & - \\
\hline Sedentary time with parents & $53(F)$ & & & $1 / 1$ & + \\
\hline Parental attitudes/beliefs towards PA & $31,31,31,31,46$ & & $31,31,34,34$ & $5 / 9$ & ?? \\
\hline Parental beliefs towards screen-based behaviours & $53(\mathrm{M})$ & & & $1 / 1$ & + \\
\hline Parental enjoyment of PA & & $17(\mathrm{~F})$ & & $1 / 1$ & - \\
\hline Parental enjoyment of screen-based behaviours & $53(F)$ & & & $1 / 1$ & + \\
\hline Parental encouragement for PA & $\mathbf{1 1 ( F )}, 45,45,46, \mathbf{5 2 ( M )}, 55,72$ & $45(F), 45,45$ & $39,45,55$ & $7 / 13$ & ?? \\
\hline Family modelling & 68,72 & & 15,25 & $2 / 4$ & $?$ \\
\hline Family support & $\begin{array}{l}\text { 18, 19, 31, 31, 31, 54, 61(M), } \\
\text { 61(M), } 72\end{array}$ & & $20,25,26,31,31,31,48$ & $9 / 16$ & $? ?$ \\
\hline Family norms & $59(\mathrm{M}), 59(\mathrm{M})$ & & 25,60 & $2 / 4$ & $?$ \\
\hline Parental barriers & & 17(F), 31 & 31 & $2 / 3$ & - \\
\hline Parental self-efficacy & & $17(F)$ & & $1 / 1$ & - \\
\hline Logistic support (e.g. paying fees, transporting children) & 31, 52(M), 52(M), 72 & & 15,31 & $4 / 6$ & ++ \\
\hline Parental perception of child's competence in PA & 4 & & 4 & $1 / 2$ & $?$ \\
\hline Parental smoking & & & 39 & $0 / 1$ & 0 \\
\hline \multicolumn{6}{|l|}{ Economic } \\
\hline Parental education & $\begin{array}{l}22,22,22,32,35(F), 38(I), 38(I I), \\
\quad 49,49,71\end{array}$ & 13(M), 22, 50 & $\begin{array}{c}2, \mathbf{2}, \mathbf{2}, \mathbf{2}, \mathbf{2}, \mathbf{2}, \mathbf{2}, 22,22,22,32,32,32 \\
33,45,45,45,46,50,50,52,66\end{array}$ & $10 / 35$ & 00 \\
\hline Household income & & & $33,45,45,45$ & $0 / 4$ & 0 \\
\hline Parental employment status & $3(F, I), 26(F), 65(F)$ & & 3(II), 32, 32, 32, 32, 45, 45, 45, 51, 57 & $3 / 13$ & 00 \\
\hline Socio-economic status & & & $23,23,37,37,37,49$ & $0 / 6$ & 00 \\
\hline
\end{tabular}


Table 3 Continued

\begin{tabular}{|c|c|c|c|c|c|}
\hline Physical activity & + & - & 0 & $n / N$ & Assoc. code \\
\hline \multicolumn{6}{|l|}{ Political } \\
\hline Parental control/supervision & $9(\mathrm{~F}), 11$ & $9,9(\mathrm{M})$ & $9,9,9$ & $2 / 7$ & 00 \\
\hline Parental rules/restriction $(\mathrm{PA})$ & $9(F), 9(F), \mathbf{1 3 ( F )}$ & $9(\mathrm{~F})$ & $9,9,9$ & $3 / 7$ & $? ?$ \\
\hline Parental rules/restriction (screen-based behaviours) & $53(\mathrm{~F})$ & $\begin{array}{c}53(\mathrm{M}), 53(\mathrm{~F}) \\
53(\mathrm{~F})\end{array}$ & & $3 / 4$ & -- \\
\hline \multicolumn{6}{|l|}{ School environment } \\
\hline \multicolumn{6}{|l|}{ Physical } \\
\hline Offering school sports & 42 & & $42,42,42$ & $1 / 4$ & 0 \\
\hline School team/school sports participation & $\mathbf{3}(\mathrm{M}, \mathrm{I}), \mathbf{3}(\mathrm{F}, \mathrm{II}), 45$ & 45 & 61,61 & $3 / 6$ & ?? \\
\hline Having a PA-promoting school environment & & & $42,42,42,42$ & $0 / 4$ & 00 \\
\hline Condition of PA-promoting school environment & 42 & & $42,42,42$ & $1 / 4$ & 0 \\
\hline Number of recreational features & & & 42,42 & $0 / 2$ & 0 \\
\hline Walking to and from school & $1,1(F), 1(F)$ & & 1 & $3 / 4$ & ++ \\
\hline \multicolumn{6}{|l|}{ Sociocultural } \\
\hline Support teacher & 26(M), 68 & & & $2 / 2$ & + \\
\hline Having class problems & & $\begin{array}{c}26(F), 26(F), \\
26(F), 26(F)\end{array}$ & & $4 / 4$ & -- \\
\hline PA with friends at school & & & 69 & $0 / 1$ & 0 \\
\hline \multicolumn{6}{|l|}{ Economic } \\
\hline School SES & $7(F), 36$ & & & $2 / 2$ & + \\
\hline \multicolumn{6}{|l|}{ Political } \\
\hline Compulsory school PA & & & 12 & $0 / 1$ & 0 \\
\hline School PA policy & & & $42,42,47$ & $0 / 3$ & 0 \\
\hline
\end{tabular}

$n$, number of studies that are related to the behaviour; $N$, number of studies that have investigated the potential correlate; Assoc. code, association code; $(F)$, association applicable only for girls; $(\mathrm{M})$, association applicable only for boys; longitudinal studies in bold; (I) and (II), analyses conducted for different time periods (e.g. different follow-ups); PA, physical activity; SES, socio-economic status.

Association code: $0=0-33 \%$ of the findings supporting the association; $00=\geq 4$ studies not finding an association; ? = indeterminate finding or $34-59 \%$ of the findings supporting the association; ? ? = $\geq 4$ studies with indeterminate findings; $+=60-100 \%$ of the findings supporting a positive association; $++=\geq 4$ studies supporting a positive association; $-=60-100 \%$ of the findings supporting a negative association; $--=\geq 4$ *PA repun by a no $13,20,28,52,71,2$

72, 75; PA reported by child: 3,55

A 
Table 4 Correlates of sedentary behaviour in 10-12-year-old children: bibliography numbers of studies reporting a positive correlation (+), a negative correlation $(-)$ or no correlation ( 0$)$ among the studies reviewed

\begin{tabular}{|c|c|c|c|c|c|}
\hline Sedentary behaviour & + & - & 0 & $n / N$ & Assoc. code \\
\hline \multicolumn{6}{|l|}{ Family environment } \\
\hline \multicolumn{6}{|l|}{ Physical } \\
\hline Number of TV in the household & $51(\mathrm{~F}), 53,74$ & & & $3 / 3$ & + \\
\hline TV in the bedroom & $53(\mathrm{~F}), 74$ & & 56,56 & $2 / 4$ & $?$ \\
\hline Presence of (cable/pay) TV & 8 & & 53 & $1 / 2$ & $?$ \\
\hline Eating in front of the TV & 30 & & & $1 / 1$ & + \\
\hline \multicolumn{6}{|l|}{ Sociocultural } \\
\hline Two-parent household & & 37, 53(F), 71(F) & 7,76 & $3 / 5$ & - \\
\hline Number of siblings & 53 & 71 & & $1 / 2$ & $?$ \\
\hline Parental ethnicity & & 8 & & $1 / 1$ & - \\
\hline Parental overweight & 71 & & & $1 / 1$ & + \\
\hline Parental sedentary time & 56,71 & & 56 & $2 / 3$ & + \\
\hline Maternal sedentary time & 53,53 & & 71 & $2 / 3$ & + \\
\hline Paternal sedentary time & 53 & & 71 & $1 / 2$ & $?$ \\
\hline Sedentary time with parents & $30,53(\mathrm{M})$ & & & $2 / 2$ & + \\
\hline Parental PA behaviour & & & 30 & $0 / 1$ & 0 \\
\hline PA with parents & & 55 & 30 & $1 / 2$ & $?$ \\
\hline Parental PA preferences & & 30 & & $1 / 1$ & - \\
\hline Parental attitude towards screen-based behaviours & & 30 & $30,30,30$ & $1 / 4$ & 00 \\
\hline Parental enjoyment of screen-based behaviours & $53(\mathrm{~F})$ & & & $1 / 1$ & + \\
\hline Parental knowledge about recommendations & & 8 & & $1 / 1$ & - \\
\hline Parental/family encouragement for PA & & & 55 & $0 / 1$ & 0 \\
\hline Having family dinners & & 74 & & $1 / 1$ & - \\
\hline Using TV as a reward & & & 53 & $0 / 1$ & 0 \\
\hline Parental activity patterns & & & 30 & $0 / 1$ & 0 \\
\hline \multicolumn{6}{|l|}{ Economic } \\
\hline Parental education & & 53,71 & 2,2 & $2 / 4$ & ?? \\
\hline Household income & & 8 & & $1 / 1$ & + \\
\hline Parental employment status & & & 51 & $0 / 1$ & 0 \\
\hline Area deprivation & & & 64 & $0 / 1$ & 0 \\
\hline Socio-economic status & & & 37 & $0 / 1$ & 0 \\
\hline \multicolumn{6}{|l|}{ Political } \\
\hline Parental rules/restriction (screen-based behaviours) & $\begin{array}{r}8,8,8,30,30,30,30,53(\mathrm{M}) \\
53(\mathrm{M}), 53(\mathrm{~F}), 53,56,56,74\end{array}$ & & $8,30,30,30,30,30,30$ & $14 / 21$ & -- \\
\hline \multicolumn{6}{|l|}{ School environment } \\
\hline \multicolumn{6}{|l|}{ Physical } \\
\hline After-school context & & & 56 & $0 / 1$ & 0 \\
\hline Economic & & & & & \\
\hline School SES & & 7 & & $1 / 1$ & - \\
\hline
\end{tabular}

$n$, number of studies that are related to the behaviour; $N$, number of studies that have investigated the potential correlate; Assoc. code, association code; $(\mathrm{F})$, association applicable only for girls; $(\mathrm{M})$, association applicable only for boys; longitudinal studies in bold; TV, television; PA, physical activity.

udies not finding an association; ? = indeterminate finding or $34-59 \%$ of the findings supporting the association; ?? = $\geq 4$ studies with indeterminate findings; $+=60-100 \%$ of the findings supporting a positive association; $-=60-100 \%$ of the findings supporting a negative association; $--=\geq 4$ studies supporting a negative association. 
Table 5 Correlates of breakfast consumption in 10-12-year-old children: bibliography numbers of studies reporting a positive correlation $(+)$, a negative correlation (-) or no correlation (0) among the studies reviewed

\begin{tabular}{|c|c|c|c|c|c|}
\hline Breakfast consumption & + & - & 0 & $n / N$ & Assoc. code \\
\hline \multicolumn{6}{|l|}{ Family environment } \\
\hline \multicolumn{6}{|l|}{ Sociocultural } \\
\hline Two-parent household & & & 67,76 & $0 / 2$ & 0 \\
\hline Parental descriptive norms & 41 & & & $1 / 1$ & + \\
\hline Parental injunctive norms & 41 & & & $1 / 1$ & + \\
\hline General parenting style & & & 67 & $0 / 1$ & 0 \\
\hline Parental permissiveness & & 67 & & $1 / 1$ & - \\
\hline Parental pressure & & & 67 & $0 / 1$ & 0 \\
\hline Parental rewards & & & 67 & $0 / 1$ & 0 \\
\hline Parental encouragement through negotiation & & & 67 & $0 / 1$ & 0 \\
\hline Parental catering on demands of children & & 67 & & $1 / 1$ & - \\
\hline Parental avoidance of negative modelling behaviour & & 67 & & $1 / 1$ & _ \\
\hline Parental verbal praise & & & 67 & $0 / 1$ & 0 \\
\hline \multicolumn{6}{|l|}{ Economic } \\
\hline Parental employment status & & & $10,24,67,67$ & $0 / 4$ & 00 \\
\hline Area deprivation & & 63 & & $1 / 1$ & - \\
\hline \multicolumn{6}{|l|}{ Political } \\
\hline Parental control/supervision & 10 & & & $1 / 1$ & + \\
\hline \multicolumn{6}{|l|}{ School environment } \\
\hline \multicolumn{6}{|l|}{ Sociocultural } \\
\hline Teacher injunctive norms & 41 & & & $1 / 1$ & + \\
\hline \multicolumn{6}{|l|}{ Economic } \\
\hline School SES & & 40 & & $1 / 1$ & - \\
\hline
\end{tabular}

$n$, number of studies that are related to the behaviour; $N$, number of studies that have investigated the potential correlate; Assoc. code, association code; SES, socio-economic status.

Association code: $0=0-33 \%$ of the findings supporting the association; $00=\geq 4$ studies not finding an association; $+=60-100 \%$ of the findings supporting a positive association; $-=60-100 \%$ of the findings supporting a negative association.

Table 6 Correlates of soft drink consumption in 10-12-year-old children: bibliography numbers of studies reporting a positive correlation $(+)$, a negative correlation $(-)$ or no correlation $(0)$ among the studies reviewed

\begin{tabular}{|c|c|c|c|c|c|}
\hline Soft drink consumption & + & - & 0 & $n / N$ & Assoc. code \\
\hline \multicolumn{6}{|l|}{ Family environment } \\
\hline \multicolumn{6}{|l|}{ Physical } \\
\hline Availability at home & $16,29,70$ & & & $3 / 3$ & + \\
\hline \multicolumn{6}{|l|}{ Sociocultural } \\
\hline Two-parent household & & & 67 & $0 / 1$ & 0 \\
\hline Parental consumption & 29 & & & $1 / 1$ & + \\
\hline General parenting style & & & 67 & $0 / 1$ & 0 \\
\hline Permissive parenting practice & 67 & & & $1 / 1$ & + \\
\hline Parental pressure & & & 67 & $0 / 1$ & 0 \\
\hline Parental rewards & & & 67 & $0 / 1$ & 0 \\
\hline Parental encouragement through negotiation & & & 67 & $0 / 1$ & 0 \\
\hline Parental catering on demands of children & & & 67 & $0 / 1$ & 0 \\
\hline Parental avoidance of negative modelling behaviour & & & 67 & $0 / 1$ & 0 \\
\hline Parental verbal praise & & & 67 & $0 / 1$ & 0 \\
\hline Having family dinners & & 27 & & $1 / 1$ & - \\
\hline \multicolumn{6}{|l|}{ Economic } \\
\hline Household income & & 21 & & $1 / 1$ & - \\
\hline Parental employment status & & 67,67 & & $2 / 2$ & _ \\
\hline \multicolumn{6}{|l|}{ Political } \\
\hline Parental limits & & 44 & & $1 / 1$ & _- \\
\hline \multicolumn{6}{|l|}{ School environment } \\
\hline \multicolumn{6}{|l|}{ Physical } \\
\hline Availability at school & 21,69 & & & $2 / 2$ & + \\
\hline Access to snack bars/competitive foods at school & & 14 & 6 & $1 / 2$ & $?$ \\
\hline Healthful school lunches & & & 6 & $0 / 1$ & 0 \\
\hline \multicolumn{6}{|l|}{ Sociocultural } \\
\hline Promotion of healthful eating at school & & & 6 & $0 / 1$ & 0 \\
\hline Participation in healthy school lunch & & 5 & & $1 / 1$ & - \\
\hline Soft drink consumption at school & 44,73 & & 16 & $2 / 3$ & + \\
\hline \multicolumn{6}{|l|}{ Economic } \\
\hline School type & & & 21 & $0 / 1$ & 0 \\
\hline
\end{tabular}

$n$, number of studies that are related to the behaviour; $N$, number of studies that have investigated the potential correlate; Assoc. code, association code; longitudinal studies in bold.

Association code: $0=0-33 \%$ of the findings supporting the association; ?=indeterminate finding or $34-59 \%$ of the findings supporting the association; $+=60-100 \%$ of the findings supporting a positive association; $-=60 \%-100 \%$ of the findings supporting a negative association. 
Parental descriptive norms, parental injunctive norms and parental control/supervision were positively related to breakfast consumption. Parental permissiveness, parental catering on demands of children, parental avoidance of negative modelling behaviour and area deprivation were inversely associated. All other variables were not associated with breakfast consumption.

School-environmental variables. Two school-environmental variables were investigated: one sociocultural and one economic environmental variable. Teacher injunctive norms was positively related and school SES was negatively related to breakfast consumption.

Generally, no studies on correlates of breakfast consumption in 10-12-year-olds have yet been conducted in North America. All studies had a cross-sectional design.

\section{Soft drink consumption (Table 6)}

Family-environmental variables. Fifteen familyenvironmental variables were studied: one physical, eleven sociocultural, two economic and one political environmental variable. Availability of soft drinks at home, parental soft drink consumption and permissive parenting style were positively related to soft drink consumption. Having family dinners, household income, parental employment status and parental limits were inversely related. The other variables were not associated with soft drink consumption.

School-environmental variables. Seven schoolenvironmental variables were investigated: three physical, three sociocultural and one economic environmental variable. Availability of soft drinks at school and soft drink consumption at school were positively associated with general soft drink consumption. Participation in healthy school lunches was inversely associated. The other variables showed an indeterminate association or no association with soft drink consumption.

Due to the low number of studies investigating correlates of soft drink consumption in this age group, no relevant comparisons could be made between European, North American and Australasian results.

\section{Discussion}

The objective of the present review was to identify familyand school-based correlates of PA, sedentary behaviour, breakfast consumption and soft drink consumption in 10-12-year-olds. To our knowledge, no review has ever investigated correlates of PA, sedentary and dietary behaviour together. The majority of the studies investigated correlates of PA behaviour, resulting in most evidence found for variables related to PA, but also in more inconsistencies between the study results. Those inconsistent results could possibly be due to methodological issues, such as the use of different instruments (child $v$. parent report, objective $v$. self-report), differences in validity and reliability of the measurements, differences in the specific sub-behaviours of PA, etc. For sedentary behaviour and especially for breakfast and soft drink consumption, few studies were available; many correlates have hardly been studied or not at all. Our study results further showed that most studies have investigated sociocultural family-environmental variables. One of the most important contributions is the specific evidence found for 10-12-year-old children. The review enables us to say with confidence that the correlates found are specifically related to EBRB of 10-12-year-olds. In previous reviews $^{(8-16)}$, a much broader age range was used, but it is clear that correlates related to health behaviour of a 6-yearold will not be similar to the correlates influencing health behaviour of a 16-year-old, for example ${ }^{(52)}$. The influence of parental behaviours varies with age ${ }^{(23)}$ and the school environment goes through significant changes in the course of the school years of a child ${ }^{(20,21)}$.

\section{Physical activity}

The most consistent evidence was found for an association of children's PA with parental/family PA, doing PA together with the parents and parental logistic support. Regarding parental PA, our results revealed that the association between mothers' and children's PA is more consistent than for fathers', suggesting mothers may be more influential for PA behaviour in this age group. There was no relevant difference by gender in the association between maternal and child PA, while the significant positive associations found between paternal and child PA mostly occurred in girls. The specific reasons why the influence of maternal and paternal PA might differ between boys and girls should be further examined. Additionally, our review demonstrated that doing physical activities together with the child is even more important, since nine out of ten studies confirmed the positive association with the child's PA level. This correlate might be less important in an older adolescent population: in a recent review ${ }^{(53)}$, parental involvement (i.e. parents doing PA with their child) was only associated with overall PA and leisure-time PA in children, not in adolescents. This emphasizes the importance of studying correlates separately for different age groups. Apart from being active role models for their children's physically active lifestyle ${ }^{(54,55)}$, parents providing logistic support might influence children's PA as well. In brief, parents play an indispensable role in PA promotion among 10-12-year-old children.

Although twelve school-environmental variables were examined, results did not yield a better understanding of the association between the school environment and children's PA behaviour. For example, having class problems was inversely related to PA, but this was only based upon one study, so cannot be considered to provide strong evidence.

\section{Sedentary bebaviour}

Most evidence was found for an inverse association between parental rules/restriction related to screen-based 
behaviours and children's actual sedentary behaviour. Encouraging parents to set rules and restrictions related to screen-based behaviours (e.g. TV or computer use) is therefore suggested as a possible strategy to reduce 10-12-year-olds' sedentary time ${ }^{(56,57)}$. Parents might consequently create limits and monitor their children's sedentary behaviour $^{(57)}$. Considering TV and computer use, children are recommended to spend no more than $2 \mathrm{~h} / \mathrm{d}$ on watching TV and using the computer or a game console ${ }^{(58)}$. Moreover, the positive association between the number of TV in the household and sedentary behaviour indicates the significant role of the home environment in influencing children's sedentary time. Given that parents have control over the acquisition of TV and computers, this offers possibilities to modify the home environment with parental assistance ${ }^{(57)}$. Furthermore, parents can be regarded as role models for sedentary behaviour, since sedentary time of the parents was positively associated with children's sedentary behaviour. Moreover, parents spending more sedentary time together with their children was related to more sedentary behaviour among children. The latter two correlates were also related to children's PA level. This accounts for parental enjoyment of screen-based behaviours and school SES as well. So despite the fact that sedentary behaviour and PA are two separate EBRB with each their own specific correlates ${ }^{(9,59)}$, some correlates were significantly related to both behaviours. These correlates are therefore considered as very important, since they are associated with two EBRB.

\section{Breakfast consumption}

Little research has been done in the field of correlates of breakfast consumption among 10-12-year-olds. Despite the limited evidence, parental descriptive and injunctive norms seemed to influence breakfast consumption in a positive way. It shows again evidence for parents as positive role models for their children. Three specific parenting practices were associated with breakfast consumption as well: parental permissiveness, parental catering on demands of the children and parental avoidance of negative modelling behaviour. Moreover, not only parents have an influence on their children's breakfast consumption, but teachers could play a role as well considering the positive relationship between teacher injunctive norms and breakfast consumption. Consequently, schools could possibly be involved in an intervention to promote breakfast consumption among children. Nevertheless, no study has ever investigated other schoolenvironmental correlates of breakfast consumption in this age group, possibly due to the fact that breakfast is an event preferably occurring at home.

\section{Soft drink consumption}

Comparable to breakfast consumption, not many studies have already examined family- and school-environmental correlates of soft drink consumption in 10-12-year-olds, but the few studies found revealed that soft drink availability at home was positively associated with soft drink consumption in three studies. As parents are primary gatekeepers of purchases at home ${ }^{(10)}$, parents could restrict soft drink availability and have a major impact upon children's soft drink consumption. Also, if soft drinks are available at home, parents might set up limits concerning soft drink consumption, since parental limits were related to soft drink consumption. Targeting these factors in an intervention programme could lead to less soft drink consumption among children. Regarding the specific parenting practices, only parental permissiveness was related to more soft drink consumption in one study and already related to less breakfast consumption as well. Parents are therefore advised to adopt a more authoritative parenting style to promote healthy behaviour among children ${ }^{(10)}$. Similar to the other EBRB, parental behaviour was once again positively related to the child's behaviour, although this was investigated by only one study.

The most important school-environmental correlates of soft drink consumption were soft drink availability and consumption at school. This strongly shows that schools can play a central role in an intervention to decrease soft drink consumption. Prohibiting soft drinks at school at that age would engender the decrease in general soft drink consumption.

\section{Limitations}

The first limitation lies within the nature of literature reviews of behavioural correlates. Identifying correlates of EBRB through a review can and should inform obesity prevention programmes to contribute to better chances of effectiveness. However, the actual mechanism is a complex web and it should be kept in mind that the present review only revealed associations between single variables and a general outcome measure (covering several specific sub-behaviours), without taking possible moderators and covariates into account. A second limitation is the possibility that not all existing studies on this topic were covered. Some articles might not be found in our databases searched or through our search strategy. The use of only English published data contributes to this limitation too. Conducting subsequent searches on specific correlates that were already identified by the first search strategy could have yielded more studies on this topic. Third, we have focused on the consistency of the association and not on the strength of the association found in primary studies. Further, conceptually similar variables were combined into a single category, even if variables were measured in a different way. Also for the behaviours, we did not differentiate between specific physical and sedentary activities, although correlates can vary depending on the specific activity ${ }^{(53)}$. Finally, most studies had a cross-sectional design through which only association could be established and not prediction or causation. Longitudinal and cross-sectional results were 
compared with each other, but no real differences were found between the results, which could be due to the low number of longitudinal studies. Future longitudinal research is needed to gain more insight into the correlates of EBRB.

\section{Conclusions}

The current review presents an overview of the studied family- and school-environmental variables. Obesity prevention programmes for this specific age group can focus on the most important modifiable correlates to change children's behaviour. Besides modifiable correlates, the review also identified non-modifiable correlates such as school SES. Such insights can help to identify specific groups 'at risk' that can be considered as important target groups for health promotion interventions. Overall, the review provides evidence for the important role that has been awarded to parents, since parental behaviour was related to children's behaviour for all four EBRB. Parents can consequently be considered as key players in the prevention of weight gain among children ${ }^{(17)}$. Interventions could help parents to create a supportive environment for their children to promote healthy behaviour ${ }^{(60)}$.

Despite all the opportunities a school can offer in health promotion, little research has been done in the field of school-based correlates of EBRB. More research is needed to focus on important school-environmental factors when developing an intervention programme.

Further, this review did not reveal relevant differences between European, North American and Australasian results. However, the number of studies in each region was often too small to make meaningful comparisons. In case of sufficient studies per region no clear differences were observed. Still little research has been executed in Europe on correlates of EBRB in 10-12-year-olds. If future studies do not contradict this finding, it can be concluded that when developing an obesity prevention programme for European schools as the ENERGY project aims to do, one can rely on non-European studies about correlates of EBRB despite the different obesity context in other continents ${ }^{(61)}$.

\section{Acknowledgements}

The ENERGY project is funded by the Seventh Framework Programme (CORDIS FP7) of the European Commission, HEALTH (FP7-HEALTH-2007-B), Grant Agreement no. 223254. The content of this article reflects only the authors' views and the European Community is not liable for any use that may be made of the information contained herein. The authors declare that they have no competing interests. J.B. developed the concept and design of the ENERGY project. M.V. conducted the systematic review with help from W.V.L., L.M. and I.D.B. M.V. wrote the first draft of the paper. All authors read and approved the final manuscript.

\section{References}

1. Lobstein T, Baur L, Uauy R et al. (2004) Obesity in children and young people: a crisis in public health. Obes Rev $\mathbf{5}$, Suppl. 5, 4-85.

2. Warschburger P (2005) The unhappy obese child. Int J Obes (Lond) 29, Suppl. 2, S127-S129.

3. Hill OJ, Wyatt HR \& Melanson EL (2000) Genetic and environmental contributions to obesity. Med Clin North Am 84, 333-346.

4. Baranowski $\mathrm{T}$ \& Jago $\mathrm{R}$ (2005) Understanding the mechanisms of change in children's physical activity programs. Exerc Sport Sci Rev 33, 163-168.

5. Brug J, Oenema A \& Ferreira I (2005) Theory, evidence and intervention mapping to improve behavior nutrition and physical activity interventions. Int J Behav Nutr Phys Act 2, 2.

6. Kremers SPJ, Visscher TLS, Seidell JC et al. (2005) Cognitive determinants of energy balance-related behaviours measurement issues. Sports Med 35, 923-933.

7. Brug J, te Velde SJ, Chinapaw MJM et al. (2010) Evidencebased development of school-based and family-involved prevention of overweight across Europe: the ENERGYproject's design and conceptual framework. BMC Public Health 10, 276.

8. Ferreira I, van der Horst K, Wendel-Vos W et al. (2006) Environmental correlates of physical activity in youth - a review and update. Obes Rev $\mathbf{8}, 129-154$.

9. Gorely T, Marshall SJ \& Biddle SJH (2004) Couch kids: correlates of television viewing among youth. Int J Behav Med 11, 152-163.

10. Patrick H \& Nicklas TA (2005) A review of family and social determinants of children's eating patterns and diet quality. J Am Coll Nutr 24, 83-92.

11. Pearson N, Biddle SJH \& Gorely T (2008) Family correlates of fruit and vegetable consumption in children and adolescents: a systematic review. Public Health Nutr 12, 267-283.

12. Pearson N, Biddle SJH \& Gorely T (2009) Family correlates of breakfast consumption among children and adolescents. Appetite 52, 1-7.

13. Rasmussen M, Krolner R, Klepp KI et al. (2006) Determinants of fruit and vegetable consumption among children and adolescents: a review of the literature. Part I: Quantitative studies. Int J Behav Nutr Phys Act 3, 22.

14. Sallis JF, Prochaska JJ \& Taylor WC (2000) A review of correlates of physical activity of children and adolescents. Med Sci Sports Exerc 32, 963-975.

15. van der Horst K, Oenema A, Ferreira I et al. (2007) A systematic review of environmental correlates of obesity-related dietary behaviors in youth. Health Educ Res 22, 203-226.

16. van der Horst K, Chin A Paw MJ, Twisk JWR et al. (2007) A brief review on correlates of physical activity and sedentariness in youth. Med Sci Sports Exerc 39, 1241-1250.

17. Golan M \& Crow S (2004) Parents are key players in the prevention and treatment of weight-related problems. Nutr Rev 62, 39-50.

18. Demory-Luce F, Morales M, Nicklas T et al. (2004) Changes in food groups consumption patterns from childhood to young adulthood: the Bogalusa Heart Study. J Am Diet Assoc 104, 1684-1691.

19. Kelder DH, Peery CL, Klepp KI et al. (1994) Longitudinal tracking of adolescent smoking, physical activity and food choice behaviors. Am J Public Health 84, 1121-1126.

20. Vereecken CA, Bobelijn K \& Maes L (2005) School food policy at primary and secondary schools in BelgiumFlanders: does it influence young people's food habits? Eur J Clin Nutr 59, 271-277.

21. Blaes A, Baquet G, Van Praagh E et al. (2011) Physical activity patterns in French youth - from childhood to adolescence - monitored with high-frequency accelerometry. Am J Hum Biol 23, 353-358. 
22. Marild S, Bondestam M, Bergström R et al. (2004) Prevalence trends of obesity and overweight among 10year-old children in western Sweden and relationship with parental body mass index. Acta Paediatr 93, 1588-1595.

23. Pugliese J \& Tinsley B (2007) Parental socialization of child and adolescent physical activity: a meta-analysis. J Fam Psychol 21, 331-343.

24. Johnson-Taylor WL \& Everhart JE (2006) Modifiable environmental and behavioral determinants of overweight among children and adolescents: report of a workshop. Obesity (Silver Spring) 14, 929-966.

25. Story M, Kaphingst KM \& French S (2006) The role of schools in obesity prevention. Future Child 16, 109-142.

26. Wechsler H, Devereaux RS, Davis M et al. (2000) Using the school environment to promote physical activity and healthy eating. Prev Med 31, Suppl. 2, S121-S137.

27. Affenito SG, Thompson DR, Barton BA et al. (2005) Breakfast consumption by African-American and white adolescent girls correlates positively with calcium and fiber intake and negatively with body mass index. $J$ Am Diet Assoc 105, 938-945.

28. Elgar FJ, Roberts C, Moore L et al. (2005) Sedentary behaviour, physical activity and weight problems in adolescents in Wales. Public Health 119, 518-524.

29. Jiménez-Pavon D, Kelly J \& Reilly JJ (2010) Associations between objectively measured habitual physical activity and adiposity in children and adolescents: systematic review. Int J Pediatr Obes 5, 3-18.

30. Marshall SJ, Gorely T \& Biddle SJH (2006) A descriptive epidemiology of screen-based media use in youth: a review and critique. $J$ Adolesc 29, 333-349.

31. Rey-Lopez JP, Vicente-Rodriguez G, Biosca M et al. (2008) Sedentary behaviour and obesity development in children and adolescents. Nutr Metab Cardiovasc Dis 18, 242-251.

32. Bachman CM, Baranowski T \& Nicklas TA (2006) Is there an association between sweetened beverages and adiposity? Nutr Rev 64, 153-174.

33. Malik VS, Schulze MB \& Hu FB (2006) Intake of sugarsweetened beverages and weight gain: a systematic review. Am J Clin Nutr 84, 274-288.

34. Douthwaite W, Summerbell CD \& Moore H (2011) Identifying the most important energy balance behaviours among 10-12 year olds, and their parents, that are associated with excessive weight gain and overweight. WP2-Phase 1 Report. http://www.projectenergy.eu/oeffentlicher_bereich/ publications/reports/WP2-Phase\%201\%20Report\%20def.pdf (accessed November 2011).

35. Kremers SP (2010) Theory and practice in the study of influences on energy balance-related behaviors. Patient Educ Couns 79, 291-298.

36. Kremers SPJ, de Bruijn GJ, Visscher TLS et al. (2006) Environmental influences on energy balance-related behaviors: a dual-process view. Int J Behav Nutr Phys Act 3, 9.

37. Swinburn B, Egger G \& Raza F (1999) Dissecting obesogenic environments: the development and application of a framework for identifying and prioritizing environmental interventions for obesity. Prev Med 29, 563-570.

38. Hinkly T, Crawford D, Salmon J et al. (2008) Preschool children and physical activity. A review of correlates. $\mathrm{Am} \mathrm{J}$ Prev Med 34, 435-441.

39. Bandura A (1986) Social Foundations of Thoughts and Action: A Social Cognitive Theory. Englewood Cliffs, NJ: Prentice Hall.

40. Ajzen I \& Fishbein M (1980) Understanding Attitudes and Predicting Social Behavior. Englewood Cliffs, NJ: Prentice Hall.

41. Bandura A (1977) A Social Learning Theory. London: Prentice Hall.

42. Welk GJ (1999) The youth physical activity promotion mode: a conceptual bridge between theory and practice. Quest 51, 5-23.
43. Kimiecik JC, Horn TS \& Shurin CS (1996) Relationships among children's beliefs, perceptions of their parents' beliefs, and their moderate-to-vigorous physical activity. Res Q Exerc Sport 67, 324-336.

44. Schultz A \& Northridge M (2004) Social determinants of health: implications for environmental health promotion. Health Educ Behav 31, 455-471.

45. Pender NJ, Walker SN, Frank-Stromborg M et al. (1990) The Health Promotion Model: Refinement and Validation Final Report. Bethesda, MD: National Center for Nursing Research.

46. Kohl HW \& Hobbs KE (1998) Development of physical activity behaviours among children and adolescents. Pediatrics 101, 549-554.

47. Sallis JF, Simons-Morton EBG, Stone EJ et al. (1992) Determinants of physical activity and interventions in youth. Med Sci Sports Exerc 24, Suppl. 6, S248-S257.

48. Stokols D (1992) Establishing and maintaining healthy environments. Towards a social ecology of health promotion. Am Psychol 47, 6-22.

49. Breslow L (1996) Social ecological strategies for promoting healthy lifestyles. Am J Health Promot 10, 253-257.

50. Eccles JS, Addler TF, Futterman R et al. (1983) Expectancies, values and academic behaviors. In Achievement and Achievement Association, pp. 75-146 [JT Spence, editor]. San Francisco, CA: Freeman.

51. Ferraro KF (1995) Fear of Crime: Interpreting Victimization Risk. Albany, NY: SUNY Press.

52. Cillero IH \& Jago R (2010) Systematic review of correlates of screen-viewing among young children. Prev Med 51, $3-10$.

53. Edwardson CL \& Gorely T (2010) Parental influences on different types and intensities of physical activity in youth: a systematic review. Psychol Sport Exerc 11, 522-535.

54. Higgins JW, Gaul C, Gibbons S et al. (2003) Factors influencing physical activity levels among Canadian youth. Can J Public Health 94, 45-51.

55. Ritchie LD, Welk G, Styne D et al. (2005) Family environment and pediatric overweight: what is a parent to do? J Am Diet Assoc 105, Suppl. 1, S70-S79.

56. Hohepa M, Scragg R, Schofield G et al. (2009) Social support for youth physical activity: importance of siblings, parents, friends and school support across a segmented school day. Int J Behav Nutr Phys Act 4, 54.

57. Granich J, Rosenberg M, Knuiman M et al. (2010) Understanding children's sedentary behaviour: a qualitative study of the family home environment. Health Educ Res 25, 199-210

58. Salmon J \& Shilton T (2004) Endorsement of physical activity recommendations for children and youth in Australia. J Sci Med Sport 7, 405-406.

59. Gordon-Larsen P, McMurray RG \& Popkin BM (2000) Determinants of adolescent physical activity and inactivity patterns. Pediatrics 105, E83.

60. Bauer KW, Neumark-Sztainer D, Fulkerson JA et al. (2011) Familial correlates of adolescent girls' physical activity, television use, dietary intake, weight, and body composition. Int J Behav Nutr Phys Act 8, 25.

61. Branca F, Nikogosian H \& Lobstein T (2007) The Challenge of Obesity in the WHO European Region and the Strategies for Response. Copenhagen: WHO Regional Ofice for Europe.

\section{Bibliography}

1. Abbot RA, Macdonald D, Nambiar S et al. (2009) The association between walking to school, daily step counts and meeting step targets in 5- to 17-year-old Australian children. Pediatr Exerc Sci 21, 520-532. 
2. Ball K, Cleland VJ, Timperio AF et al. (2009) Socioeconomic position and children's physical activity and sedentary behaviors: longitudinal findings from the CLAN study. J Phys Act Health 6, 289-298.

3. Barnett TA, O'Loughlin J \& Paradis G (2002) One- and two-year predictors of decline in physical activity among inner-city schoolchildren. Am J Prev Med 23, 121-128.

4. Bois JE, Sarrazin PG, Brustad RJ et al. (2005) Elementary schoolchildren's perceived competence and physical activity involvement: the influences of parents' role modelling behaviours and perceptions of their child's competence. Psychol Sport Exerc 6, 381-397.

5. Briefel RR, Wilson A \& Gleason PM (2009) Consumption of low-nutrient, energy-dense foods and beverages at school, home, and other locations among school lunch participants and nonparticipants. J Am Diet Assoc 109, Suppl. 2, S79-S90.

6. Briefel RR, Crepinsek MK, Cabili C et al. (2009) School food environments and practices affect dietary behaviors of US public school children. J Am Diet Assoc 109, Suppl. 2, S91-S107.

7. Brodersen NH, Steptoe A, Boniface DR et al. (2007) Trends in physical activity and sedentary behavior in adolescence: ethnic and socioeconomic differences. Br J Sports Med $\mathbf{4 1}$, 140-144.

8. Carlson SA, Fulton JE, Lee SM et al. (2010) Influence of limit-setting and participation in physical activity on youth screen time. Pediatrics 126, E89-E96.

9. Carver A, Timperio A, Hesketh K et al. (2010) Are children and adolescents less active if parents restrict their physical activity and active transport due to perceived risk? Soc Sci Med 70, 1799-1805.

10. Cheng TSY, Tse LA, Yu ITS et al. (2008) Children's perceptions of parental attitude affecting breakfast skipping in primary sixth-grade students. J Sch Health 78, 203-208.

11. Cleland V, Timperio A, Salmon J et al. (2010) Predictors of time spent outdoors among children: 5-year longitudinal findings. J Epidemiol Community Health 64, 400-406.

12. Cleland V, Dwyer T, Blizzard L et al. (2008) The provision of compulsory school physical activity: associations with physical activity, fitness and overweight in childhood and twenty years later. Int J Behav Nutr Phys Act 5, 14.

13. Crawford D, Cleland V, Timperio A et al. (2010) The longitudinal influence of home and neighbourhood environments on children's body mass index and physical activity over 5 years: the CLAN study. Int J Obes (Lond) $\mathbf{3 4}$, 1177-1187.

14. Cullen KW \& Zakeri I (2004) Fruits, vegetables, milk and sweetened beverages consumption and access to à la carte/ snack bar meals at school. Am J Public Health 94, 463-467.

15. Davison KK \& Jago R (2009) Change in parent and peer support across ages 9 to $15 \mathrm{yr}$ and adolescent girls' physical activity. Med Sci Sports Exerc 41, 1816-1825.

16. Denney-Wilson E, Crawford D, Dobbins T et al. (2009) Influences on consumption of soft drinks and fast foods in adolescents. Asia Pac J Clin Nutr 18, 447-452.

17. DiLorenzo TM, Stucky-Ropp RC, Van der Wal JS et al. (1998) Determinants of exercise among children. II. A longitudinal analysis. Prev Med 27, 470-477.

18. Dishman RK, Dunn AL, Sallis JF et al. (2010) Socialcognitive correlates of physical activity in a multi-ethnic cohort of middle-school girls: two-year prospective study. $J$ Pediatr Psychol 35, 188-198.

19. Dollman J \& Lewis NR (2009) Interactions of socioeconomic position with psychosocial and environmental correlates of children's physical activity: an observational study of South Australian families. Int J Behav Nutr Phys Act 6, 56.

20. Duncan SC, Duncan TE, Strycker LA et al. (2007) A cohortsequential latent growth model of physical activity from ages 12 to 17 years. Ann Behav Med 33, 80-89.
21. Fernandes MM (2008) The effect of soft drink availability in elementary schools on consumption. J Am Diet Assoc 108, $1445-1452$.

22. Franzini L, Elliott MN, Cuccaro P et al. (2009) Influences of physical and social neighborhood environments on children's physical activity and obesity. Am J Public Health 99, 271-278.

23. Freitas D, Maia J, Beunen G et al. (2007) Socio-economic status, growth, physical activity and fitness: The Madeira Growth Study. Ann Hum Biol 34, 107-122.

24. Gaina A, Sekine M, Chandola T et al. (2009) Mother employment status and nutritional patterns in Japanese junior high schoolchildren. Int J Obes (Lond) 33, 753-757.

25. Garcia AW, Broda MAN, Frenn M et al. (1995) Gender and developmental differences in exercise beliefs among youth and prediction of their exercise behavior. $J$ Sch Health $\mathbf{6 5}$, 213-219.

26. Gillander Gådin K \& Hammarström A (2002) Can schoolrelated factors predict future health behaviour among young adolescents? Public Health 116, 22-29.

27. Gillman MW, Rifas-Shiman SL, Fraizer L et al. (2000) Family dinner and diet quality among older children and adolescents. Arch Fam Med 9, 235-240.

28. Griffith JR, Clasey JL, King JT et al. (2007) Role of parents in determining children's physical activity. World J Pediatr 3 , 265-270.

29. Grimm GC, Harnack L \& Story M (2004) Factors associated with soft drink consumption in school-aged children. $J$ Am Diet Assoc 104, 1244-1249.

30. He M, Piché L, Beynon C et al. (2010) Screen-related sedentary behaviors: children's and parents' attitudes, motivations, and practices. J Nutr Edu Behav 42, 17-25.

31. Heitzler CD, Martin SL, Duke J et al. (2006) Correlates of physical activity in a national sample of children aged 9-13 years. Prev Med 42, 254-260.

32. Hesketh K, Graham M \& Waters E (2008) Children's afterschool activity: associations with weight status and family circumstance. Pediatr Exerc Sci 20, 84-94.

33. Huang S, Hung W, Sharpe PA et al. (2010) Neighborhood environment and physical activity among urban and rural schoolchildren in Taiwan. Health Place 16, 470-476.

34. Kahn JA, Huang B, Gillman MW et al. (2008) Patterns and determinants of physical activity in US adolescents. $J$ Adolesc Health 42, 369-377.

35. Kimm SYS, Glynn NW, Kriska AM et al. (2002) Decline in physical activity in black girls and white girls during adolescence. $N$ Engl J Med 347, 709-715.

36. Kolle E, Steene-Johannessen J, Klasson-Heggebø L et al. (2009) A 5-yr change in Norwegian 9-yr-olds' objectively assessed physical activity level. Med Sci Sports Exerc 41, 1368-1373.

37. Lindquist CH, Reynolds KD \& Goran MI (1999) Sociocultural determinants of physical activity among children. Prev Med 29, 305-312.

38. Madsen KA, McCulloch CE \& Crawford PB (2009) Parent modeling: perceptions of parents' physical activity predict girls' activity throughout adolescence. J Pediatr 154, 278-283.

39. McMinn AM, van Sluijs EMF, Wedderkopp N et al. (2008) Sociocultural correlates of physical activity in children and adolescents: findings from the Danish arm of the European Youth Heart Study. Pediatr Exerc Sci 20, 319-332.

40. Moore GF, Tapper K, Murphy S et al. (2007) Associations between deprivation, attitudes towards eating, breakfast and breakfast eating behaviours in 9-11-year-olds. Public Health Nutr 10, 582-589.

41. Moore GF, Moore L \& Murphy S (2009) Normative and cognitive correlates of breakfast skipping in 9-11-year-old schoolchildren in Wales. Appetite 53, 332-337.

42. Nichol ME, Pickett W \& Janssen I (2009) Associations between school recreational environments and physical activity. J Sch Health 79, 247-254. 
43. Nichols-English GJ, Lemmon CR, Litaker MS et al. (2006) Relations of black mothers' and daughters' body fatness, physical activity beliefs and behavior. Ethn Dis 16, 172-179.

44. Nickelson J, Roseman MG \& Forthofer MS (2003) Associations between parental limits, school vending machine purchases, and soft drink consumption among Kentucky middle school students. J Nutr Educ Behav 42, 115-122.

45. O'Loughlin J, Paradis G, Kishchuk N et al. (1999) Prevalence and correlates of physical activity behaviors among elementary schoolchildren in multiethnic, low income, inner-city neighborhoods in Montreal, Canada. Ann Epidemiol 9, 397-407.

46. Panter JR, Jones AP, van Sluijs EMF et al. (2010) Attitudes, social support and environmental perceptions as predictors of active commuting behaviour in school children. $J$ Epidemiol Community Health 64, 41-48.

47. Panter JR, Jones AP, van Sluijs EMF et al. (2010) Neighborhood, route, and school environments and children's active commuting. Am J Prev Med 38, 268-278.

48. Pate RR, Trost SG, Felton GM et al. (1997) Correlates of physical activity in rural youth. Res Q Exerc Sport 68, 241-248.

49. Piko BF \& Keresztes N (2008) Sociodemographic and socioeconomic variations in leisure time physical activity in a sample of Hungarian youth. Int J Public Health 53, 306-310.

50. Riddoch CJ, Mattocks C, Deere K et al. (2007) Objective measurement of levels and patterns of physical activity. Arch Dis Child 92, 963-969.

51. Roemmich JN, Epstein LH, Raja S et al. (2007) The neighborhood and home environments: disparate relationships with physical activity and sedentary behaviors in youth. Ann Behav Med 33, 29-38.

52. Sallis JF, Alcaraz JE, McKenzie TL et al. (1999) Predictors of change in children's physical activity over 20 months variations by gender and level of adiposity. Am J Prev Med 16, 222-229.

53. Salmon J, Timperio A, Telford A et al. (2005) Association of family environment with children's television viewing and with low level of physical activity. Obes Res 13, 1939-1951.

54. Saunders RP, Pate RR, Felton GM et al. (1997) Development of questionnaires to measure psychosocial influences on children's physical activity. Prev Med 26, 241-247.

55. Springer AE, Kelder SH \& Hoelscher DM (2006) Social support, physical activity and sedentary behavior among 6th-grade girls: a cross-sectional study. Int J Behav Nutr Phys Act 3, 8.

56. Springer AE, Kelder SH, Barroso CR et al. (2010) Parental influences on television watching among children living on the Texas-Mexico border. Prev Med 51, 112-117.

57. Timperio A, Crawford D, Telford A et al. (2003) Perceptions about the local neighborhood and walking and cycling among children. Prev Med 38, 39-47.

58. Timperio A, Ball K, Salmon J et al. (2006) Family, social, and environmental correlates of active commuting to school. Am J Prev Med 30, 45-51.

59. Trost SG, Pate RR, Ward DS et al. (1999) Determinants of physical activity in active and low-active, sixth grade African-American youth. J Sch Health 69, 29-33.

60. Trost SG, Pate RR, Ward DS et al. (1999) Correlates of objectively measured physical activity in preadolescent youth. Am J Prev Med 17, 120-126.
61. Trost SG, Pate RR, Saunders R et al. (1997) A prospective study of the determinants of physical activity in rural fifthgrade children. Prev Med 26, 257-263.

62. Unger JB, Reynolds K, Shakib S et al. (2004) Acculturation, physical activity, and fast-food consumption among AsianAmerican and Hispanic adolescents. J Community Health 29, 467-481.

63. Utter J, Scragg R, Mhurchu $\mathrm{CN}$ et al. (2007) At-home breakfast consumption among New Zealand: associations with body mass index and related nutrition behaviors. $J \mathrm{Am}$ Diet Assoc 107, 570-576.

64. Utter J, Scragg R \& Schaaf D (2006) Associations between television viewing and consumption of commonly advertised foods among New Zealand children and young adolescents. Public Health Nutr 9, 606-612.

65. Van Lenthe FJ, Boreham CA, Twisk JWR et al. (2001) Socioeconomic position and coronary heart disease risk factors in youth - findings from the Young Hearts Project in Northern Ireland. Eur J Public Health 11, 43-50.

66. van Sluijs EMF, Skidmore PML, Mwanza K et al. (2008) Physical activity and dietary behaviour in a populationbased sample of British 10-year old children: the SPEEDY study (Sport, Physical activity and Eating behaviour: Environmental Determinants in Young people). BMC Public Health 8, 388.

67. Vereecken C, Legiest E, De Bourdeaudhuij I et al. (2009) Associations between general parenting styles and specific food-related parenting practices and children's food consumption. Am J Health Promot 23, 233-240.

68. Villard LC, Rydén L \& Stahle A (2007) Predictors of healthy behaviours in Swedish school children. Eur J Cardiovasc Prev Rehabil 14, 366-372.

69. Voorhees CC, Murray D, Welk G et al. (2005) The role of peer social network factors and physical activity in adolescent girls. Am J Health Behav 29, 183-190.

70. Vue H \& Reicks M (2007) Individual and environmental influences on intake of calcium-rich food and beverages by young Hmong adolescent girls. J Nutr Educ Behav 39, 264-272.

71. Wagner A, Klein-Platat C, Arveiler D et al. (2004) Parent-child physical activity relationships in 12 -year old French students do not depend on family socioeconomic status. Diabetes Metab 30, 359-366.

72. Welk GJ, Wood K \& Morss G (2003) Parental influences on physical activity in children: an exploration of potential mechanisms. Pediatr Exerc Sci 15, 19-33.

73. Wiecha JL, Finkelstein D, Troped PJ et al. (2006) School vending machine use and fast-food restaurant use are associated with sugar-sweetened beverage intake in youth. J Am Diet Assoc 106, 1624-1630.

74. Wiecha JL, Sobol AM, Peterson KE et al. (2001) Household television access: associations with screen time, reading, and homework among youth. Ambul Pediatr 1, 244-251.

75. Wrotniak BH, Zimmer N, Dingle A et al. (2007) Physical activity, health, and dietary patterns of middle school children. Pediatr Phys Ther 19, 203-210.

76. Yannakoulia M, Papanikolaou K, Hatzopoulou I et al. (2008) Association between family divorce and children's BMI and meal patterns: The GENDAI study. Obesity (Silver Spring) 16, 1382-1387. 